\title{
Cervical spinal cord atrophy
}

An early marker of progressive MS onset

Burcu Zeydan, MD, Xinyi Gu, MD, Elizabeth J. Atkinson, MS, B. Mark Keegan, MD, Brian G. Weinshenker, MD, Jan-Mendelt Tillema, MD, Daniel Pelletier, MD, Christina J. Azevedo, MD, Christine Lebrun-Frenay, MD, Aksel Siva, MD, Darin T. Okuda, MD, Kejal Kantarci, MD, MS, and Orhun H. Kantarci, MD

Neurol Neuroimmunol Neuroinflamm March 2018;5:e435. doi:10.1212/NXI.0000000000000435

\section{Abstract}

\section{Objective}

To assess whether cervical spinal cord atrophy heralds the onset of progressive MS.

\section{Methods}

We studied 34 individuals with radiologically isolated syndrome (RIS) and 31 patients with relapsing-remitting MS (RRMS) age matched to 25 patients within a year of onset of secondary progressive MS (SPMS). Two raters independently measured (twice per rater) the cervical spinal cord average segmental area (CASA) $\left(\mathrm{mm}^{2}\right)$ of axial T2-weighted images between C2 and C7 landmarks. The midsagittal T2-weighted image from the end of C2 to the end of C7 vertebra was used to measure the cervical spine (c-spine) length $(\mathrm{mm})$. Sex, age at cervical MRI, number and location of cervical spinal cord lesions, c-spine length, and diagnoses were analyzed against the outcome measures of CASA and C2 and C7 slice segmental areas.

\section{Results}

Intrarater and interrater agreement was excellent (intraclass correlation coefficient $>0.97)$. The CASA area $(p=0.03)$ and C7 area $(p=0.002)$ were smaller in SPMS compared with RRMS. The C2 area $(p=0.027)$, CASA $(p=0.004)$, and C7 area $(p=0.003)$ were smaller in SPMS compared with RIS. The C2 area did not differ between SPMS and RRMS $(p=0.09)$. The C2 area $(p=0.349)$, CASA $(p=0.136)$, and C7 area $(p=0.228)$ did not differ between RIS and MS (SPMS and RRMS combined). In the multivariable model, $\geq 2$ cervical spinal cord lesions were associated with the C2 area $(p=0.008)$, CASA $(p=0.009)$, and C7 area independent of disease course $(p=0.017)$. Progressive disease course was associated with the $\mathrm{C} 7$ area independent of the cervical spinal cord lesion number $(p=0.004)$.

\section{Conclusion}

Cervical spinal cord atrophy is evident at the onset of progressive MS and seems partially independent of the number of cervical spinal cord lesions.

\section{Classification of evidence}

This study provides Class III evidence that MRI cervical spinal cord atrophy distinguishes patients at the onset of progressive MS from those with RIS and RRMS.

\author{
Correspondence to \\ Dr. Kantarci \\ kantarci.orhun@mayo.edu
}




\section{Glossary}

CASA = cervical spinal cord average segmental area; c-spine $=$ cervical spine; CIS $=$ clinically isolated syndrome; PPMS = primary progressive MS; RIS = radiologically isolated syndrome; RRMS = relapsing-remitting MS; SAPMS = single-attack progressive MS; SPMS = secondary progressive MS.

Semiautomated and automated methods for quantifying cervical spinal cord atrophy in MS have previously been described for analysis of head MRI (only upper cervical cord) or dedicated cervical spinal cord MRI. ${ }^{1-22}$ Cervical spinal cord atrophy occurs early in MS and even in those with clinically isolated syndrome (CIS), ${ }^{4,15,23}$ but is most prominent in established progressive MS. ${ }^{1-3,5,6,8,9,11-14,17}$ The cervical spinal cord area correlates inversely with disability in MS. ${ }^{1-13,20}$

Previous studies included control individuals, patients with relapsing-remitting MS (RRMS), and predominantly patients with long-standing progressive disease course (progressive disease duration of $\geq 10$ years). ${ }^{1,3,6,7,9,11,19}$ Studies that focused on early progressive MS included patients with only primary progressive MS (PPMS) ${ }^{17,21,22}$ It is not established whether cervical spinal cord atrophy is present at the onset of the progressive phase in secondary progressive MS (SPMS).

Bout-onset progressive MS refers to patients with progressive MS who had clinically evident relapses before the onset of progressive disease course and consists of 2 categories: SPMS and single-attack progressive MS (SAPMS) as previously described. ${ }^{24}$ In this study, we group patients with SAPMS together with SPMS. As opposed to PPMS, SPMS reflects a biological and clinical continuum from CIS or RRMS, therefore presenting an opportunity to study a comparative group to determine whether spinal cord atrophy is restricted to or highly associated with the onset of the symptomatic progressive phase in MS. Similarly, RRMS can evolve from radiologically isolated syndrome (RIS); therefore, presenting an opportunity to determine whether spinal cord atrophy is restricted to or highly associated with evolution of symptomatic MS.

We compared cervical spinal cord areas at the onset of symptomatic progressive MS of patients with SPMS with agematched patients with RRMS who had not developed progressive MS at the time of their cervical MRI acquisition as well as individuals with RIS who had not developed symptomatic MS at the time of their cervical MRI acquisition.

\section{Methods}

In this study (Class III evidence), we tested the specific hypothesis that cervical spinal cord atrophy heralds the onset of progressive MS by comparing sex and age-group-matched patients at the onset of progressive MS with RRMS without progression and individuals with RIS who are asymptomatic.

\section{Study population}

We identified our index population of patients with SPMS from a previously described large clinic-based and populationbased study cohort. ${ }^{24}$ We included patients with SPMS who had available cervical spinal cord MRIs within 1 year of progressive MS onset (figure). We established the age limits and distribution of the available cervical MRIs in the SPMS group.

We then identified patients who remained as having RRMS at the time of last follow-up from the same cohort. ${ }^{24}$ We matched the patients from this group to those from the SPMS group by the cervical spinal cord MRI age limits and distribution. We finally identified individuals who remained as having RIS who had cervical spinal cord MRIs within the age limits of RRMS and patients with SPMS from a previously established cohort. ${ }^{25}$

Diagnoses were confirmed with the most recent diagnostic criteria. $^{26,27}$ Our study, while cross-sectional in nature, assumes that the patients included represent a diagnostic continuum as having RIS can evolve to CIS or RRMS, and CIS or RRMS can evolve to SPMS. It was a priori decided to exclude patients with PPMS for 2 reasons: (1) since PPMS can evolve from RIS $^{25}$ but cannot evolve from RRMS, inclusion of these patients would break the uniform clinical continuum assumption as described above and (2) since by definition, clinical progression should have been present for over 1 year for PPMS diagnosis to be established, and these patients more commonly are seen for the first time outside this 1-year period; cervical MRIs from these patients would not strictly represent the earliest changes closest to the onset of clinically evident progressive MS.

\section{Standard protocol approvals, registrations, and patient consents}

The study protocol was approved by the institutional review board, and written informed consent was signed by every participant.

\section{MRI acquisition and inclusion criteria}

Since study scans were originally acquired as part of routine clinical evaluations, once the clinically matched groups were established, we used additional MRI inclusion and exclusion criteria pertaining to available cervical MRIs to select the final study group where all patients had to have (1) 1.5T MRIs as a standard for cervical spinal cord imaging representing reallife application, (2) sagittal and axial T2-weighted images, and (3) $\leq 4 \mathrm{~mm}$ slice thickness. Scans were excluded (1) if they did not fulfill the inclusion criteria, (2) if patients had significant scoliosis or kyphosis to impair measurements, and (3) if the MRI quality as subjectively assessed by 2 of the investigators 

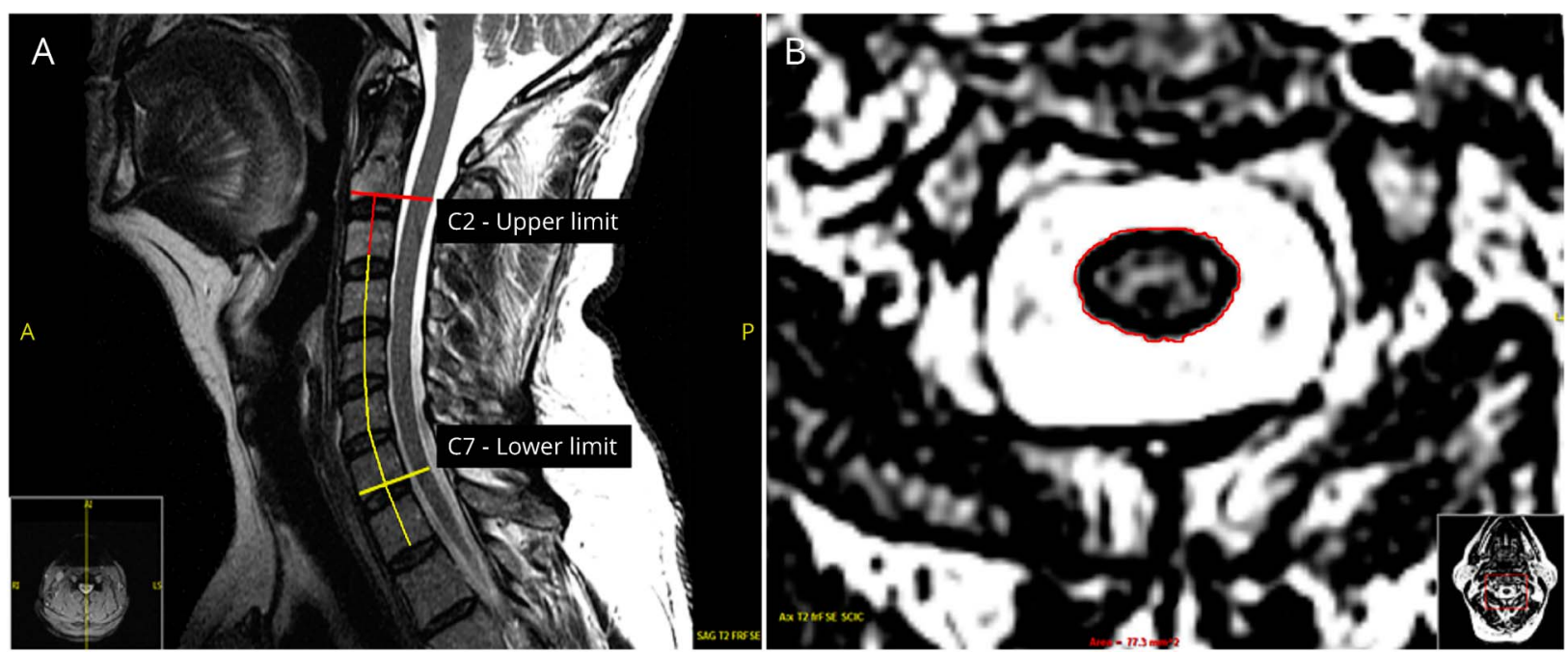

(A) C-spine length: first, the midsagittal T2-weighted image was identified. After adjustments for contrast and edge sharpness, the top of the C2-C3 disc space was established as the upper limit. The top of the C7-T1 disc space was established as the lower limit. A vertical line was drawn to connect each vertebra from the highest point in the disc space to the next. The 6 vertical line measurements from the end of C2 to the end of C7 vertebra were summed up to calculate the c-spine length ( $\mathrm{mm}$ ). (B) Cervical spinal cord area: T2-weighted axial MRIs were used for measuring the C2 cross-sectional area, C7 cross-sectional area, and CASA. To draw 2 horizontal lines perpendicular to the mid-spine vertical line at the upper limit and at the lower limit, the top of the C2-C3 disc space and the top of the C7-T1 disc space described in A were used. After the upper and lower limits of the axial images were identified, adjustments for contrast and edge sharpness were made. Then, these landmarks were used to measure the uppermost (C2) and lowermost (C7) $\mathrm{cross}^{-5 e c t i o n a l ~ a r e a s ~(m m ²) . ~ A l l ~ a v a i l a b l e ~}$ axial slices between these limits were measured and averaged for calculating CASA $\left(\mathrm{mm}^{2}\right)$. CASA = cervical spinal cord average segmental area; c-spine = cervical spine.

(B.Z. and X.G.) (no movement or pulsation artifacts, etc.) was redeemed low. Each included MRI was also given an additional subjective quality score that was later used in the multivariable models to assess any independent effect on our results. When possible, studies were restricted to the Mayo Clinic for uniformity, but this was not used as absolute criteria for inclusion, and group differences were assessed to ensure that there was no systematic difference between groups.

\section{MRI analyses}

A fully manual approach was used (QREADS Clinical Image Viewer, version 5.9.2.1, copyright 2017, Mayo Clinic). Two investigators blinded to diagnoses (B.Z. and X.G.) independently measured the cervical spine (c-spine) length and cervical spinal cord areas: C2 cord area, C7 cord area, and whole cervical spinal cord average segmental area (CASA) from $\mathrm{C} 2$ to $\mathrm{C} 7$. An initial training set of 10 patients was used to assess intraindividual and interindividual variability. A second set of 10 patients was used to minimize variability further. Intrarater and interrater reliability were assessed using intraclass correlations and found to be all $>0.97$ for the $\mathrm{C} 2$ cord area, C7 cord area, and CASA. For the final analyses, a conservative approach was used, as for every measurement, the intrarater and interrater variabilities were further minimized by averaging twice replicated measurements per rater.

\section{C-spine length measurement}

After adjusting for contrast and edge sharpness, we measured the length of the cervical spinal cord from the top of the $\mathrm{C} 2-\mathrm{C} 3$ interspace to the top of the C7-T1 interspace on midsagittal T2-weighted MRIs. Each intervertebral segment was measured by an axial mid-vertebral line, and all 6 measurements from the end of $\mathrm{C} 2$ to the end of $\mathrm{C} 7$ vertebra were summed up to calculate the c-spine length $(\mathrm{mm})$ (figure, $\mathrm{A})$. If the c-spine was not well aligned or had a curvature (e.g., mild scoliosis), 3 consecutive center sagittal $\mathrm{T} 2$-weighted sequences were measured from the end of $\mathrm{C} 2$ to the end of $\mathrm{C} 7$ vertebra, following the same steps and averaged for the c-spine length. The c-spine length measurement was used in the final multivariable model.

\section{Cervical spinal cord area measurement}

After adjusting for contrast and edge sharpness, an outline of the spinal cord border was marked on each $\mathrm{T} 2$-weighted axial image slice between the upper (C2) and lower (C7) limits established by a horizontal line intersecting the mid-spine vertical line at $90^{\circ}$ at the upper and lower limits as described above (figure, A and B). The C2 cross-sectional area, C7 cross-sectional area, and CASA calculated by averaging all segmental areas between $\mathrm{C} 2$ and $\mathrm{C} 7$ limits were used as outcomes in the final analyses.

\section{Cervical spinal cord lesion assessment}

Sagittal and axial T2-weighted images were used. Cervical spinal cord lesions from $\mathrm{C} 2$ to $\mathrm{C} 7$ were marked and confirmed independently by 2 investigators (B.Z. and O.H.K.). The lesions were counted, and their locations were identified according to anatomical spinal cord columns of anterolateral, 
posterior, or both. The lesion number and location were used in the final multivariable model.

\section{Statistical analyses}

Group differences were assessed using Student's $t$ test for continuous data, and the $\chi^{2}$ test for categorical data. Pearson correlation coefficients were used to summarize relationships between the age and the various measures of the cervical spinal cord area. Linear regression models predicting the area were fit using the independent variables sex, age at cervical MRI, subjective MRI quality score, number and location of cervical spinal cord lesions, c-spine length, and diagnosis. Model assumptions were checked, including a check for highly influential observations, non-normality, and nonlinearity. All analyses were run using software R, version 3.3.1.

\section{Results}

\section{Demographic and clinical characteristics}

The study population consisted of 90 patients: 34 individuals with RIS, 31 patients with RRMS, and 25 patients with SPMS (4 SAPMS and 21 SPMS) fulfilled all inclusion and exclusion criteria (table). In the whole group, $71 \%$ were women, and the mean age $( \pm S D)$ at cervical MRI was $47.4 \pm 11.1$ years. The proportion of women among RIS, RRMS, and SPMS groups was similar $(p=0.898)$. Age (mean $\pm \mathrm{SD})$ at cervical MRI in the SPMS group was $49.1 \pm 9.3$ years and in the age-matched RRMS group was $49.0 \pm 10.3$ years $(p=0.95)$. Age at cervical MRI in the RIS group (mean \pm SD; $44.8 \pm 12.7$ years) while slightly younger did not differ from patients with MS ( $p=$ 0.078). MS disease duration at the time of cervical MRI in SPMS (mean \pm SD; $12.9 \pm 7.2$ years) was shorter than the RRMS group (mean \pm SD; $19.0 \pm 11.6$ years $)(p=0.03)$.

\section{Imaging characteristics}

As expected, men had taller c-spine (mean $\pm \mathrm{SD}$; $90.0 \pm 6.1$ $\mathrm{mm})$ than women $($ mean $\pm \mathrm{SD}$; $80.9 \pm 4.1 \mathrm{~mm})(p<0.001)$ and slightly but not significantly larger C2 area (men: $80.5 \pm$ $11.1 \mathrm{~mm}^{2}$, women: $\left.79.6 \pm 13.9 \mathrm{~mm}^{2}, p=0.755\right)$, C7 area (men: $63.2 \pm 10.4 \mathrm{~mm}^{2}$, women: $61.8 \pm 10.3 \mathrm{~mm}^{2}, p=0.552$ ), and CASA (men: $80.3 \pm 8.9 \mathrm{~mm}^{2}$, women: $77.6 \pm 11.1 \mathrm{~mm}^{2}, p=$ 0.269 ). Within the age limits of our study, age at cervical MRI did not correlate with the $\mathrm{C} 2$ area $(r=0.01, p=0.90)$, C7 area $(r=0.18, p=0.08)$, or CASA $(r=0.04, p=0.69)$.

Overall, the C2 area, CASA, and C7 area were larger in RIS than those in the RRMS and SPMS groups (table). However,

Table Demographic and imaging characteristics

\begin{tabular}{|c|c|c|c|c|c|c|}
\hline & \multirow[b]{2}{*}{ RIS ( $n=34)$} & \multicolumn{4}{|c|}{ MS } & \multirow[b]{2}{*}{$p$ Value $^{b}$} \\
\hline & & RRMS (n = 31) & SPMS $(n=25)$ & Total $(n=56)$ & $p$ Value $^{a}$ & \\
\hline Women, n (\%) & $25(74)$ & $22(71)$ & $17(68)$ & $39(70)$ & $0.99^{c}$ & $0.877^{c}$ \\
\hline MS duration at cervical MRI, $y$, mean \pm SD & NA & $19.0 \pm 11.6$ & $12.9 \pm 7.2$ & $16.2 \pm 10.3$ & $0.03^{d}$ & NA \\
\hline Progressive MS duration at cervical MRI, $y$, mean \pm SD & NA & NA & $0.4 \pm 0.5$ & NA & NA & NA \\
\hline Age at cervical MRI, mean \pm SD & $44.8 \pm 12.7$ & $49.0 \pm 10.3$ & $49.1 \pm 9.3$ & $49.0 \pm 9.8$ & $0.95^{d}$ & $0.078^{\mathrm{d}}$ \\
\hline \multicolumn{7}{|l|}{ Cervical spinal cord area, $\mathrm{mm}^{2}$, mean $\pm \mathrm{SD}$} \\
\hline C2 & $81.3 \pm 10.2$ & $81.4 \pm 16.8$ & $75.2 \pm 10.1$ & $78.6 \pm 14.4$ & $0.11^{d}$ & $0.349^{d}$ \\
\hline CASA & $80.4 \pm 8.7$ & $79.8 \pm 12.6$ & $73.5 \pm 8.9$ & $77.0 \pm 11.5$ & $0.04^{d}$ & $0.136^{d}$ \\
\hline C7 & $63.7 \pm 9.8$ & $64.8 \pm 10.9$ & $56.0 \pm 8.8$ & $60.9 \pm 10.8$ & $0.002^{d}$ & $0.228^{d}$ \\
\hline \multicolumn{7}{|l|}{ Cervical spinal cord lesion number } \\
\hline Median (range) & $0(0-4)$ & $2(0-6)$ & $3(0-8)$ & $2(0-8)$ & $0.19^{d}$ & $<0.001^{\mathrm{d}}$ \\
\hline$\geq 1$ lesion $(\%)$ & $15(44)$ & $27(87)$ & $24(96)$ & $51(91)$ & $0.49^{c}$ & $<0.001^{c}$ \\
\hline$\geq 2$ lesions $(\%)$ & $4(12)$ & $19(61)$ & $18(72)$ & $37(66)$ & $0.58^{c}$ & $<0.001^{c}$ \\
\hline \multicolumn{7}{|l|}{ Cervical spinal cord lesion location (\%) } \\
\hline Anterolateral columns & $8(53)$ & $9(33)$ & $9(38)$ & $18(35)$ & & \\
\hline Posterior columns & $1(7)$ & $1(4)$ & $2(8)$ & $3(6)$ & $0.71^{c}$ & $0.423^{c}$ \\
\hline Both & $6(40)$ & $17(63)$ & $13(54)$ & $30(59)$ & & \\
\hline
\end{tabular}

Abbreviations: CASA = cervical spinal cord average segmental area; NA = not applicable; RIS = radiologically isolated syndrome; RRMS = relapsing-remitting MS; SPMS = secondary progressive MS.

a RRMS VS SPMS.

${ }^{b}$ RIS vS MS.

${ }^{c} \chi^{2}$ test.

d'Student's $t$ test. 
there was no difference at the C2 level between RIS and MS $(p=0.349)$ or between RRMS and SPMS $(p=0.11)$. The CASA did not differ between RIS and MS $(p=0.136)$, but was smaller in SPMS than RRMS $(p=0.04)$. The C7 area did not differ between RIS and MS $(p=0.228)$, but was smaller in SPMS compared with RRMS $(p=0.002)$. RIS differed most from SPMS in the C2 area $(p=0.027)$, CASA $(p=0.004)$, and C7 area $(p=0.003)$.

Overall, the MS group more frequently had cervical cord lesions $(91 \%)$ than the RIS $(44 \%)$ group $(p<0.001)$. However, SPMS within 1 year of progressive MS onset and agematched RRMS groups did not differ in the proportion of cervical cord lesions $(p=0.19)$ (table). For the whole group, purely posterior column lesions were observed less commonly than purely anterolateral lesions (table).

Multivariable model's fit predicting cervical spinal cord area measurements included the independent variables sex, age at cervical MRI, number and location of cervical spinal cord lesions, c-spine length, diagnosis, and subjective MRI quality metric. After adjusting for the other model variables, having $\geq 2$ lesions remained as an independent determinant of the $\mathrm{C} 2$ area $(\beta=-9.5, p=0.008)$, CASA $(\beta=-9.0, p=0.009)$, and C7 area $(\beta=-7.6, p=0.017)$. After adjusting for the other model variables including lesion number, having SPMS (as opposed to RRMS) remained as an independent determinant of a smaller $C 7$ area $(\beta=7.6, p=0.004)$, but not the $C 2$ area $(\beta=$ $4.2, p=0.163)$. The other metrics including subjective quality score did not have an independent association with the outcomes studied $(p>0.05)$.

\section{Discussion}

In this cross-sectional study, patients with progressive MS had established cervical spinal cord atrophy within 1 year of the onset of the clinically detectable progressive phase compared with age-matched patients with RRMS and RIS. This suggests that cervical spinal cord atrophy is a potential biomarker for early progressive disease to discriminate those with RRMS without progression and individuals with RIS who are asymptomatic.

However, our study has limitations. We selected patients with RRMS from a population-based cohort where these patients had already been established as not having developed SPMS by longitudinal follow-up as previously published. ${ }^{24}$ By age matching their available cervical MRIs to established SPMS patients' first year cervical MRIs, we were able to study whether atrophy heralds the beginning of progressive disease course in MS. Although the disease duration of the RRMS group was longer than the preprogressive relapsing phase of the SPMS group, the RRMS group still had the larger average cervical spinal cord area than the SPMS group. Since we selected patients with RRMS who have not developed SPMS after longitudinal clinical follow-up to be able to match the RRMS cervical MRI age to the onset of clinical SPMS, we had to use historical MRIs rather than a prospective standardized MRI set. While the latter would be optimal, based on SPMS evolution rates, ${ }^{24}$ such a study would require an average of 15 years or longer of follow-up with standardized MRIs from the onset of RRMS, which was not feasible for our study construct. As it stands, our study should be seen as a supportive basis for such a long-term expensive effort rather than as conclusive evidence.

Previous studies showed that patients with long-standing progressive MS (SPMS, PPMS, or both) had smaller cervical spinal cord areas compared with healthy controls or patients with RRMS. ${ }^{1-3,5,6,8,9,11-14,17}$ Several studies, which evaluated the early disease course of progressive MS, included only PPMS ${ }^{17,21,22}$ and by the classic definition of PPMS, the studied patients could not have been within 1 year of the disease onset. Whereas the patients we included in our study were at the onset of their SPMS. As cervical spinal cord atrophy was also shown to further worsen longitudinally with progressive disease course, ${ }^{6,11,16} 1$ logical deduction from our study is that prominent spinal cord atrophy may start during the relapsing-remitting phase of the disease for patients who will later develop clinical signs of progressive MS. We did not specifically assess the rate of spinal cord atrophy before the onset of progressive MS, which is a limitation of our cross-sectional study design. Longitudinal studies in RRMS could directly address whether a previous increase in the rate of spinal cord atrophy during the RRMS phase differentiated the patients who would develop SPMS. Despite technical challenges in multicenter implementation, our study points out the importance of monitoring asymptomatic spinal cord disease as a harbinger of progressive MS onset potentially as a more relevant biomarker than total brain atrophy. It would be interesting to assess the timing of such a shift in the rate of spinal cord atrophy for neuroprotection trials in the RRMS phase.

By including individuals with RIS who remained asymptomatic at the time of their cervical spinal cord MRI in the study, we were able to demonstrate that the spinal cord areas did not significantly differ between RRMS and RIS groups, despite the fact that the RIS group was slightly younger than the MS group. At the time of cervical MRI, individuals with RIS had 3 factors previously associated with a lower likelihood of MS development; they were predominantly women, had lower spinal cord lesion load, and on average were much older than 37 years. ${ }^{25}$ The largest difference in cervical spinal cord atrophy was observed between RIS and SPMS groups. As the RRMS group's cervical MRI areas resembled the asymptomatic RIS group more than the SPMS group, this finding further suggested that early prominent cervical spinal cord volume loss heralds progressive MS.

The presence of cervical spinal cord atrophy in MS compared with healthy controls was shown before. ${ }^{1,3,10}$ Our current construct focused on testing the hypothesis that early progressive MS would have more spinal cord atrophy than agematched patients with RIS and RRMS and therefore did not require a healthy control group. A future study comparing 
healthy controls, mechanical spinal cord injury, RIS, and MS may provide further insights on whether cervical spinal cord atrophy (like thalamic atrophy ${ }^{28}$ ) starts already at the RIS phase.

We studied the CASA from C2 to C7 rather than methods commonly evaluating the upper cervical cord area ${ }^{3-6,14,18,20}$ or cervical cord cross-sectional area of different anatomical levels such as C2, C5, or between C2 and C5. ${ }^{1,7-9,11,12,15}$ The upper cervical cord is commonly chosen because of technical feasibility of image acquisition (this segment is included in brain MRIs), easier spinal cord segmentation, ${ }^{14,29}$ and the potential for lower amount of motion artifacts. ${ }^{3}$ Although the crosssectional area approach based on the upper cervical cord segments is very reproducible, it does not provide a total estimate of the whole cervical spinal cord damage $e^{13,21,22,30}$ or assess cranial-caudal gradient in spinal cord volume loss. The whole cervical spinal cord was evaluated only in a few studies. $^{2,13}$ We found that the C7 segmental area had the greatest difference between the RRMS and SPMS groups, while the CASA had less of a difference and the $\mathrm{C} 2$ segmental area had the least difference. We could not further fine tune this segmental differentiation without standardized MRIs and could not specifically study the thoracic spinal cord to confirm this further (most patients did not have thoracic spinal cord scans obtained at the time of cervical scans). Nevertheless, spinal cord atrophy in early progressive MS seemingly is more prominent caudally, a result that needs to be confirmed in other studies.

In our study, the frequency of cervical spinal cord lesions in the whole study cohort was relatively low compared with other studies, ${ }^{3,31,32}$ likely because we also included individuals with RIS who are less likely to have spinal cord lesions than established MS. The highest cervical lesion frequency was found in the SPMS group, compatible with previous qualitative and quantitative cervical spinal cord lesion load assessments in progressive MS., ${ }^{9}$ Cervical spinal cord atrophy was associated with the number of cervical spinal cord lesions in a previous study. ${ }^{3}$ A different study showed that spinal cord atrophy was independent of the presence of cervical lesions. ${ }^{1}$ Previous pathology studies have demonstrated that individual lesions play a minor role in local atrophy. ${ }^{33,34}$ We found that having $\geq 2$ cervical spinal cord lesions was associated with cervical spinal cord area measurements independent of diagnoses, but also segmental area loss happened independent of lesion load, especially in the caudal cervical spinal cord. Our study further supports the previous conclusions that cumulative mechanisms other than just focal demyelination (e.g., Wallerian degeneration) may be responsible for spinal cord atrophy in progressive MS. ${ }^{1,35,36}$

While a manual method as ours applicable to real-world clinical data of various sources may be used as a proof of concept and is the gold standard in developing semiautomated or automated methods, ${ }^{1-22}$ it cannot be used beyond limited sample size studies because of the laborintensive nature of the analysis. As such, this is a limitation of our study, and any large-scale application of our findings in MS (e.g., for clinical trials) will require semiautomated or automated methods validated against manual measurements. ${ }^{8,9,37}$ In the same token, dedicated volumetric scans (not available to us) as opposed to axial and sagittal T2-weighted scans could provide more robust quantification for semiautomated or automated methods for large-scale future prospective studies.

We recognize another limitation of our study. While we accounted for the possible independent effect of sex, age at cervical MRI, number and location of cervical spinal cord lesions, c-spine length, and diagnosis in our multivariable models, we did not account for other parameters such as brain lesion volume simply because the nonstandard image collection did not allow for available head MRIs for all patients at the same time as their cervical MRIs. Several normalization strategies for cord measurements such as intracranial volume, cervical spinal cord lesions, and spinal cord length were previously evaluated, ${ }^{2,4,13,38,39}$ whereas some studies did not use any normalization methods ${ }^{3,14}$ due to the rationale of these factors having a low ability to improve discrimination between groups. ${ }^{3,13}$ Inconsistent results were also reported about the correlation between the spinal cord area and the number of brain lesions or brain atrophy. ${ }^{4,36}$ We believe that brain abnormalities including cortical or deep gray matter as well as white matter atrophy and lesions themselves may still lead to secondary effects in the spinal cord, independent of spinal cord lesions through Wallerian degeneration as our study would also suggest. Given the lack of standardized brain MRIs, we were not able to analyze the contribution of brain pathology on spinal cord atrophy in the current study construct. Any future longitudinal study should account for this possibility of both distal (i.e., thoracic spinal cord) and proximal (i.e., brain, brain stem, and cerebellar) CNS lesions on cervical spinal cord atrophy.

\section{Author Contributions}

B.Z.: study concept and design, acquisition of data, and analysis and interpretation. X.G.: acquisition of data. E.J.A.: analysis and interpretation. B.M.K., B.G.W., J.-M.T., D.P., C.J. A., C.L.-F., A.S., and D.T.O.: critical revision of the manuscript for important intellectual content. K.K.: analysis and interpretation and study supervision. O.H.K.: study concept and design, acquisition of data, analysis and interpretation, and study supervision.

\section{Study Funding}

No targeted funding reported.

\section{Disclosure}

B. Zeydan received support from the Turkish Neurological Society. X. Gu reports no disclosures. E.J. Atkison received research support from Biogen and NIA. B.M. Keegan serves on the editorial board of Multiple Sclerosis and Related Disorders; receives publishing royalties from Cambridge 
University Press; consulted for Novartis, Bristol-Myers Squibb, and Bioness; and receives research funding from Biogen Pharmaceuticals. B.G. Weinshenker served on the scientific advisory board of Novartis and Mitsubishi; served on the editorial board of Canadian Journal of Neurological Sciences, Turkish Journal of Neurology, and Neurology ${ }^{\circledR}$; holds patent for and receives royalties from a NMO-IG for diagnosis of neuromyelitis optica; consulted for Caladrius and BrainStorm Cell Therapeutics; is an adjudication committee member of MedImmune pharmaceuticals and Alexion Pharmaceuticals; and received research support from the GuthyJackson Charitable Foundation. J.-M. Tillema receives research support from the NCATS/NIH. D. Pelletier consulted for Biogen, EMD Serono, Sanofi Genzyme, Novartis, Hoffman La Roche, and Actelion and received research support from the National Multiple Sclerosis Society. C.J. Azevedo served on the scientific advisory board of Genzyme, Guerbet, Genentech, and Biogen and received research support from the University of Southern California Clinical and Translational Science Institute and the Race to Erase MS Foundation. C. Lebrun-Frenay served on the scientific advisory board of Biogen, Novartis, Merck, Genzyme, TEVA, and Roche; received travel funding and/or speaker honoraria from Biogen, Merck, Genzyme, and Roche; served on the editorial board of Revue Neurologique; and received research support from the French MS Society (SFSEP) EDMUS (OFSEP Center) Foundation. A. Siva received travel funding from Merck Serono, Biogen Idec/Gen Pharma of Turkey, Novartis, Sanofi Genzyme, Roche, and TEVA; served on the editorial board of Journal of the Neurological Sciences, Journal of Headache and Pain, and Turkish Neurological Journal; consulted for Biogen IDEC, Novartis, Merck Serono, Bayer, TEVA, Sanofi Genzyme, and Roche; served on the speakers' bureau of EXCEMED, Sanofi Genzyme, Merck Serono, Biogen Idec/ Gen Pharma of Turkey, and TEVA; and received research support from The Scientific and Technological Research Council of Turkey. D.T. Okuda received travel funding and speaker honoraria from Acorda Therapeutics, Genentech, Genzyme, and TEVA; holds patents for Methods, Apparatuses, and Systems for Creating 3-Dimensional Representations Exhibiting Geometric and Surface Characteristics of Brain Lesions, System and method for medical surveillance through personal communication device; consulted for Celgene, Bayer, EMD Serono, Genzyme, and Novartis; and served on the speakers' bureau of Acorda Therapeutics, Genzyme, and TEVA. K. Kantarci served on the data safety monitoring board of Takeda Global Research \& Development Center, Pfizer Inc., and Janssen Alzheimer Immunotherapy and received research support from the NIH and Minnesota Partnership for Biotechnology and Medical Genomics. O.H. Kantarci received speaker honoraria (paid to Mayo Clinic) from Novartis and Biogen; performed a grant review for The National Multiple Sclerosis Society; and received research support from Biogen, the Multiple Sclerosis Society, the Mayo Foundation, and the Hilton Foundation. Go to Neurology.org/nn for full disclosure forms.
Received May 24, 2017. Accepted in final form November 28, 2017.

\section{References}

1. Rocca MA, Horsfield MA, Sala S, et al. A multicenter assessment of cervical cord atrophy among MS clinical phenotypes. Neurology 2011;76:2096-2102.

2. Healy BC, Arora A, Hayden DL, et al. Approaches to normalization of spinal cord volume: application to multiple sclerosis. J Neuroimaging 2012;22:e12-e19.

3. Daams M, Weiler F, Steenwijk MD, et al. Mean upper cervical cord area (MUCCA) measurement in long-standing multiple sclerosis: relation to brain findings and clinical disability. Mult Scler 2014;20:1860-1865.

4. Biberacher V, Boucard CC, Schmidt P, et al. Atrophy and structural variability of the upper cervical cord in early multiple sclerosis. Mult Scler 2015;21:875-884.

5. Lukas C, Sombekke MH, Bellenberg B, et al. Relevance of spinal cord abnormalities to clinical disability in multiple sclerosis: MR imaging findings in a large cohort of patients. Radiology 2013;269:542-552.

6. Lukas C, Knol DL, Sombekke MH, et al. Cervical spinal cord volume loss is related to clinical disability progression in multiple sclerosis. J Neurol Neurosurg Psychiatry 2015;86:410-418.

7. Yiannakas MC, Mustafa AM, De Leener B, et al. Fully automated segmentation of the cervical cord from T1-weighted MRI using PropSeg: application to multiple sclerosis. Neuroimage Clin 2016;10:71-77.

8. Horsfield MA, Sala S, Neema M, et al. Rapid semi-automatic segmentation of the spinal cord from magnetic resonance images: application in multiple sclerosis. Neuroimage 2010;50:446-455.

9. Kidd D, Thorpe JW, Thompson AJ, et al. Spinal cord MRI using multi-array coils and fast spin echo. II. Findings in multiple sclerosis. Neurology 1993;43:2632-2637.

10. Losseff NA, Webb SL, O'Riordan JI, et al. Spinal cord atrophy and disability in multiple sclerosis. A new reproducible and sensitive MRI method with potential to monitor disease progression. Brain 1996;119:701-708.

11. Stevenson VL, Leary SM, Losseff NA, et al. Spinal cord atrophy and disability in MS: a longitudinal study. Neurology 1998;51:234-238.

12. Kearney H, Altmann DR, Samson RS, et al. Cervical cord lesion load is associated with disability independently from atrophy in MS. Neurology 2015;84:367-373.

13. Zivadinov R, Banas AC, Yella V, Abdelrahman N, Weinstock-Guttman B, Dwyer MG Comparison of three different methods for measurement of cervical cord atrophy in multiple sclerosis. AJNR Am J Neuroradiol 2008;29:319-325.

14. Liu Y, Lukas C, Steenwijk MD, et al. Multicenter validation of mean upper cervical cord area measurements from head 3D T1-weighted MR imaging in patients with multiple sclerosis. AJNR Am J Neuroradiol 2016;37:749-754.

15. Brex PA, Leary SM, O'Riordan JI, et al. Measurement of spinal cord area in clinically isolated syndromes suggestive of multiple sclerosis. J Neurol Neurosurg Psychiatry 2001;70:544-547.

16. Furby J, Hayton T, Altmann D, et al. A longitudinal study of MRI-detected atrophy in secondary progressive multiple sclerosis. J Neurol 2010;257:1508-1516.

17. Bieniek M, Altmann DR, Davies GR, et al. Cord atrophy separates early primary progressive and relapsing remitting multiple sclerosis. J Neurol Neurosurg Psychiatry 2006;77:1036-1039.

18. Mann RS, Constantinescu CS, Tench CR. Upper cervical spinal cord cross-sectional area in relapsing remitting multiple sclerosis: application of a new technique for measuring cross-sectional area on magnetic resonance images. J Magn Reson Imaging 2007;26:61-65.

19. Kearney H, Miszkiel KA, Yiannakas MC, Altmann DR, Ciccarelli O, Miller DH. Grey matter involvement by focal cervical spinal cord lesions is associated with progressive multiple sclerosis. Mult Scler 2016;22:910-920.

20. Lin X, Tench CR, Turner B, Blumhardt LD, Constantinescu CS. Spinal cord atrophy and disability in multiple sclerosis over four years: application of a reproducible automated technique in monitoring disease progression in a cohort of the interferon beta-1a (Rebif) treatment trial. J Neurol Neurosurg Psychiatry 2003; 74:1090-1094.

21. Stevenson VL, Miller DH, Leary SM, et al. One year follow up study of primary and transitional progressive multiple sclerosis. J Neurol Neurosurg Psychiatry 2000;68: 713-718.

22. Ingle GT, Stevenson VL, Miller DH, Thompson AJ. Primary progressive multiple sclerosis: a 5-year clinical and MR study. Brain 2003;126:2528-2536.

23. Brownlee WJ, Altmann DR, Alves Da Mota P, et al. Association of asymptomatic spinal cord lesions and atrophy with disability 5 years after a clinically isolated syndrome. Mult Scler 2017;23:665-674

24. Tutuncu M, Tang J, Zeid NA, et al. Onset of progressive phase is an age-dependent clinical milestone in multiple sclerosis. Mult Scler 2013;19:188-198.

25. Okuda DT, Siva A, Kantarci O, et al. Radiologically isolated syndrome: 5-year risk for an initial clinical event. PLoS One 2014;9:e90509.

26. Okuda DT, Mowry EM, Beheshtian A, et al. Incidental MRI anomalies suggestive of multiple sclerosis: the radiologically isolated syndrome. Neurology 2009;72:800-805.

27. Polman $\mathrm{CH}$, Reingold SC, Banwell B, et al. Diagnostic criteria for multiple sclerosis: 2010 revisions to the McDonald criteria. Ann Neurol 2011;69:292-302.

28. Azevedo CJ, Overton E, Khadka S, et al. Early CNS neurodegeneration in radiolog ically isolated syndrome. Neurol Neuroimmunol Neuroinflamm 2015;2:e102. doi: 10.1212/NXI.0000000000000102.

29. Klein JP, Arora A, Neema M, et al. A 3T MR imaging investigation of the topography of whole spinal cord atrophy in multiple sclerosis. AJNR Am J Neuroradiol 2011;32: $1138-1142$. 
30. Rashid W, Davies GR, Chard DT, et al. Increasing cord atrophy in early relapsingremitting multiple sclerosis: a 3 year study. J Neurol Neurosurg Psychiatry 2006;77:51-55.

31. Weier K, Mazraeh J, Naegelin Y, et al. Biplanar MRI for the assessment of the spinal cord in multiple sclerosis. Mult Scler 2012;18:1560-1569.

32. Bot JC, Barkhof F, Polman CH, et al. Spinal cord abnormalities in recently diagnosed MS patients: added value of spinal MRI examination. Neurology 2004;62:226-233.

33. Evangelou N, DeLuca GC, Owens T, Esiri MM. Pathological study of spinal cord atrophy in multiple sclerosis suggests limited role of local lesions. Brain 2005; 128:29-34.

34. Bergers E, Bot JC, De Groot CJ, et al. Axonal damage in the spinal cord of MS patients occurs largely independent of T2 MRI lesions. Neurology 2002;59:1766-1771.
35. Lin X, Tench CR, Evangelou N, Jaspan T, Constantinescu CS. Measurement of spinal cord atrophy in multiple sclerosis. J Neuroimaging 2004;14:20S-26S.

36. Bot JC, Barkhof F. Spinal-cord MRI in multiple sclerosis: conventional and nonconventional MR techniques. Neuroimaging Clin N Am 2009;19:81-99.

37. Kearney H, Yiannakas MC, Abdel-Aziz K, et al. Improved MRI quantification of spinal cord atrophy in multiple sclerosis. J Magn Reson Imaging 2014;39:617-623.

38. Song F, Huan Y, Yin H, et al. Normalized upper cervical spinal cord atrophy in multiple sclerosis. J Neuroimaging 2008;18:320-327.

39. Oh J, Seigo M, Saidha S, et al. Spinal cord normalization in multiple sclerosis. J Neuroimaging 2014;24:577-584. 


\section{Neurology \\ Neuroimmunology \& Neuroinflammation}

Cervical spinal cord atrophy: An early marker of progressive MS onset

Burcu Zeydan, Xinyi Gu, Elizabeth J. Atkinson, et al.

Neurol Neuroimmunol Neuroinflamm 2018;5;

DOI 10.1212/NXI.0000000000000435

This information is current as of January 22, 2018

\section{Updated Information \& Services}

References

Citations

Subspecialty Collections

Permissions \& Licensing

Reprints including high resolution figures, can be found at: http://nn.neurology.org/content/5/2/e435.full.html

This article cites 39 articles, 9 of which you can access for free at: http://nn.neurology.org/content/5/2/e435.full.html\#\#ref-list-1

This article has been cited by 5 HighWire-hosted articles: http://nn.neurology.org/content/5/2/e435.full.html\#\#otherarticles

This article, along with others on similar topics, appears in the following collection(s):

MRI

http://nn.neurology.org//cgi/collection/mri

Multiple sclerosis

http://nn.neurology.org//cgi/collection/multiple_sclerosis

Information about reproducing this article in parts (figures,tables) or in its entirety can be found online at:

http://nn.neurology.org/misc/about.xhtml\#permissions

Information about ordering reprints can be found online: http://nn.neurology.org/misc/addir.xhtml\#reprintsus

Neurol Neuroimmunol Neuroinflamm is an official journal of the American Academy of Neurology.

Published since April 2014, it is an open-access, online-only, continuous publication journal. Copyright

Copyright $\left({ }^{\circ} 2018\right.$ The Author(s). Published by Wolters Kluwer Health, Inc. on behalf of the American Academy of Neurology.. All rights reserved. Online ISSN: 2332-7812.

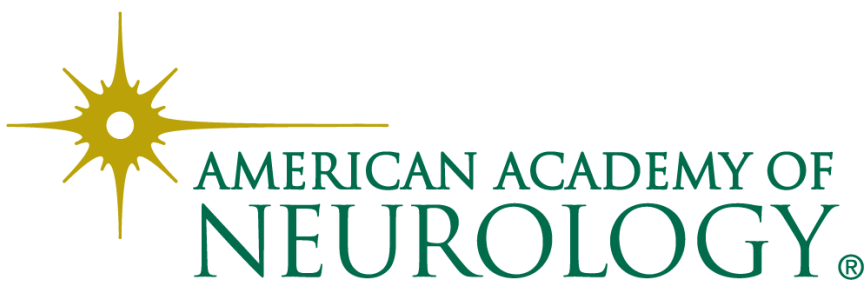

